\title{
Transluminal Attenuation Gradient for the Noninvasive Assessment of Functional Significance in Coronary Artery Stenoses
}

\author{
Marius Orzan¹, Alexandra Stănescu², Tiberiu Nyulas, Mirabela Morariu², Nora Rat', Sebastian \\ Condrea $^{2}$, Imre Benedek ${ }^{1,2}$ \\ 1 University of Medicine and Pharmacy, Tîrgu Mureș, Romania \\ 2 Center of Advanced Research in Multimodality Cardiac Imaging, Cardio Med Medical Center, Tîrgu Mureș, Romania
}

\section{CORRESPONDENCE}

\section{Alexandra Stănescu}

Str. 22 Decembrie 1989 nr. 76

540124 Tîrgu Mureș, Romania Tel:

+40265217333

E-mail: alexandrastanescu90@gmail.com

\section{ARTICLE HISTORY}

Received: 20 October, 2016

Accepted: 3 December, 2016
Marius Orzan • Str. Gheorghe Marinescu nr. 38 540139 Tîrgu Mureș, Romania. Tel: +40 265215551. E-mail: orzan_marius@yahoo.com

Tiberiu Nyulas • Str. Gheorghe Marinescu nr. 38 540139 Tîrgu Mureș, Romania. Tel: +40 265215551. E-mail: tiberiu.nyulas@gmail.com

Mirabela Morariu • Str. 22 Decembrie 1989 nr. 76 540124 Tîrgu Mures, Romania. Tel: +40 265217333. E-mail: mirabela.morariu@yahoo.com

Nora Rat • Str. Gheorghe Marinescu nr. 38, 540139 Tîrgu Mures, Romania. Tel: +40 265215 551. E-mail: ratnora@gmail.com

Sebastian Condrea • Str. 22 Decembrie 1989 nr. 76 540124 Tîrgu Mureș, Romania. Tel: +40 265217333. E-mail: sebastian.condrea@yahoo.com

Imre Benedek • Str. Gheorghe Marinescu nr. 38 540139 Tîrgu Mureș, Romania. Tel: +40 265215551.

E-mail: imrebenedek@yahoo.com

\begin{abstract}
Invasive and noninvasive methods currently used for imaging-based evaluation of the coronary tree reveal a high number of moderate degree coronary artery stenoses, and the decision to revascularize or not such lesions could be difficult in different clinical settings. Therefore, the need for evaluating the functional significance of such lesions appeared obvious and led to the introduction of fractional flow reserve methodology, a new tool proposed for the evaluation of the functional impact of a coronary stenosis. At the same time, new tools have been proposed for the same application, such as the transluminal attenuation gradient along a coronary artery stenosis, determined using cardiac computed angiography. The aim of this short update is to summarize the recent advances in the field of invasive and noninvasive evaluation of the functional significance of coronary artery stenoses.
\end{abstract}

Keywords: attenuation gradient, coronary artery stenosis, fractional flow reserve

\section{BACKGROUND}

Cardiovascular diseases are in present one of the major causes of death and morbidity, and a large amount of public expenses in the healthcare sector are dedicated to the treatment of these devastating diseases. At the moment, the trend in the assessment of the severity of coronary artery diseases is oriented towards noninvasive methods, the classic angiography remaining the first option mainly for emergency cases. Currently cardiac computed tomography angiography (CCTA) is the method of choice for the noninvasive evaluation of coronary plaques, being able to characterize stable and unstable plaques according to their specific features such as plaque burden, percent of stenosis, remodeling index, and contrast density. ${ }^{1}$

Several invasive imaging methods such as intravascular ultrasound (IVUS), optical coherence tomography (OCT) or near-infrared spectroscopy (NIRS) 
have been proposed as useful tools for the assessment of coronary lesions during invasive procedures performed in the catheterization laboratory.

IVUS associated with virtual histology is the invasive method that evaluates the entire thickness of the arterial wall using ultrasounds. The different components of the plaque defined by virtual histology (the necrotic core, fibrotic tissue, fibrofatty tissue and dense calcium) are color-coded in real time, showing the content of the atherosclerotic plaque and contributing to the identification and understanding of the vulnerabilization phenomenon that occurs at this level.2,3 It is also useful for the identification of the vulnerable plaque and for the determination of stent dimensions prior to the stenting procedure.

Optical Coherence Tomography (OCT) is an optical equivalent of IVUS and has ten times better resolution. ${ }^{4}$ Since its introduction, it rapidly became the most frequently used technique in the imagistic evaluation of the coronary arteries.

OCT can distinguish tissue features into calcified, lipidrich plaque or fibrous tissue, and can detect the thin-cap fibroatheroma. OCT systems require the elimination of blood while the examination is performed, to avoid the high attenuation of light by blood. The new platforms allow real-time 3D visualization of the OCT evaluation, which can be superimposed in multi-imagistic systems with coronarography evaluation, thus presenting advantages in case of coronary revascularization. OCT is also used in identifying post-interventional complications regarding the stent implantation: dissection, thrombus formation and partial stent apposition or stent strut coverage (at long-time follow-up after implantation).

Near-infrared spectroscopy is a technique used for the evaluation of atheromatous plaque vulnerability, based on the evaluation of the lipid content of the plaque. It provides a chemogram of the arterial wall, by precise identification of lipid-rich plaques.

All these invasive imagistic evaluation techniques are able to identify the presence of coronary stenoses, without giving clear information on the relevance of the stenosis and its hemodynamic impact. Therefore, they are less useful in cases when there was not a clear indication for revascularization. Given that the functional significance of borderline coronary stenoses proved to be a useful parameter in grading the severity of coronary stenoses, the need to evaluate the functional importance of such lesions appeared obvious and led to the introduction of fractional flow reserve methodology, a new tool proposed for the assessment of functional impact of a coronary lesion.
Fractional flow reserve is a derivative of arterial pressure measuring, which evaluates the functional significance of the stenoses. This invasive method measures the ratio of maximum blood flow in a stenotic artery to normal maximal flow. The distal value is obtained using the FFR catheter, while the aortic reference value is obtained using the coronary guide catheter. A ratio under 0.8 identifies a hemodynamically significant stenosis. Studies have shown the role of myocardial revascularization in ischemia-inducing lesions, namely lesions with the index below 0.8 , whereas the revascularization of lesions with an index above 0.8 has not proved any benefits regarding survival or the outcomes. ${ }^{5-7}$

The routine evaluation of FFR decreased the rates of all types of adverse events by almost $30 \%$. The use of FFR reduces the complication incidence probably by decreasing the number of implanted stents, through the more rational use of stents. FFR is frequently used in patients with multivascular lesions, but it has proven its utility in all patients. ${ }^{8-10}$

\section{CCTA AND TRANSLUMINAL ATTENUATION GRADIENT}

CCTA, with its application for FFRCT, represents the noninvasive alternative for the estimation of the functional significance of a coronary lesion, being currently under investigation for validation. CCTA is currently the most used investigation in ambulatory settings, associating very small risks and a minimum dose of contrast.

Computed tomography allows the classification of patients in a cardiovascular risk group considering certain parameters such as the Calcium Scoring. ${ }^{10}$ At the same time, this method can reveal the presence of certain parameters associated with vulnerable coronary plaques, such as spotty calcifications, burden with a low-density plaque and active remodeling. ${ }^{11,12}$ However, conventional computed tomography is useful in the exclusion of coronary stenoses, but it is limited by the poor image quality in some cases and by a smaller diameter of the arteries. ${ }^{13}$ Other limitations of CCTA are represented by the difficulty to visualize the coronary lumen in cases with extensive calcifications, as well as in patients with atrial fibrillation or frequent arrhythmias. However, the recently introduced systems with 256 slices allow the exploration of atrial fibrillation patients, as well as patients presenting ventricular arrhythmias.

At the moment, it is considered that multi-imaging hybrid platforms, such as PET-CT, offer significant advantages regarding patient management, as they provide infor- 
mation simultaneously concerning the coronary anatomy, the myocardial perfusion as well as the necrosis..$^{14,15}$

CCTA imaging for the evaluation of functional significance estimation comprises quantitative plaque investigation, such as aggregated plaque dimensions, transluminal attenuation gradient, myocardial CT perfusion, and FFR$\mathrm{CT}$. The integration of these new technologies increases the diagnostic performance in the identification of myocardial ischemia. ${ }^{16,17}$

However, in several groups of patients (i.e. multivascular patients, which frequently present significant calcifications), CCTA is not able to provide a clear verdict concerning the revascularization indication. Therefore, new applications for assessing the functional signification of the coronary stenosis by using computed tomography were developed.

Such a new application is represented by the transluminal attenuation gradient (TAG) along a coronary stenosis, the coefficient of linear regression between luminal attenuation and axial distance from the coronary ostium. FFRCT was calculated from CCTA images with the use of computational fluid dynamics.

TAG evaluation is currently performed manually, and ideally it is recommended that the exploration should be completed during a single cardiac contraction in order to objectively evaluate the concentration difference along the artery or between two arteries.

The use of 320-row CT allowed the evaluation of the coronary vascular system during a single cardiac beat, using specialized software to automatically measure the contrast gradient. Studies have shown that there are no significant differences regarding the manual and the automatic evaluation, the time of examination being reduced by $68 \%$ in the automatic method. ${ }^{2,9}$

Another evaluation method of the functional significance of coronary stenosis, related with TAG, is the corrected coronary opacification (CCO). This method measures the difference between the contrast density concentration in the artery and the descendent thoracic aorta at the same level.

Studies have shown that the measurement of contrast gradient through CT has a lower specificity than the angiographic FFR. ${ }^{18,19}$ Studies conducted using 320-slice devices with specialized software emphasize the importance of functional evaluation and showed that the evaluation of functional significance is superior to the simple tomography evaluation. ${ }^{20}$

The attenuation of the luminal gradient in the coronary vessel is represented by the modification of attenuation on CT examination (Hounsfield unit; HU) on a distance of 10 $\mathrm{mm}$ in the coronary vessel (unit: $\mathrm{HU} / 10 \mathrm{~mm}$ ), and is calculated as a linear regression between the $\mathrm{CT}$ attenuation and the length after the ostium. ${ }^{16}$

The optimum TAG cut-off value corresponding to the 0.8 FFR index is still under debate, but it has been demonstrated that if a significant stenosis is present, the slope of the diagram attained from the attenuation gradient will present a descending tendency, because the contrast attenuation becomes reduced in the distal arterial region. ${ }^{21,22}$

However, TAG is limited not only by the evaluation during a single cardiac beat, but also by the direction and type of scanning, as well as the timing differences, and although the initial results obtained with TAG were promising, at the moment it can be used especially to assess the functional degree and route of collateral vessels in subjects with total chronic coronary occlusions.

\section{CONCLUSIONS}

In conclusion, TAG is a useful new tool developed for evaluating the functional significance of coronary artery lesions, which requires further assessment in order to replace the classic FFR.

\section{CONFLICT OF INTEREST}

Nothing to declare.

\section{ACKNOWLEDGEMENT}

This study was supported via the research grant no. 103545/2016, contract number 43/05.09.2016 , entitled "High performance multimodal MRI/CT imaging platform, for applications in computational medicine, nanoparticles and hybrid imaging for the research of atherothrombotic disorders - CARDIO IMAGE", financed by the Romanian Ministry of European Funds, the Romanian Government and the European Union.

\section{REFERENCES}

1. Benedek T, Gyöngyösi M, Benedek I. Multislice computed tomographic coronary angiography for quantitative assessment of culprit lesions in acute coronary syndromes. Can J Cardiol. 2013;29:364-371.

2. Chatzizisis YS, George E, Cai T, et al. Accuracy and reproducibility of automated, standardized coronary transluminal attenuation gradient measurements. Int J Cardiovasc Imaging. 2014;30:1181-1189.

3. König A, Klauss V. Virtual histology. Heart. 2007;93:977-982.

4. Terashima M, Kaneda H, Suzuki T. The Role of Optical Coherence Tomography in Coronary Intervention. The Korean Journal of Internal Medicine. 2012;27:1-12.

5. Wongpraparut N, Yalamanchili V, Pasnoori V, et al. Thirty-month outcome after fractional flow reserve-guided versus conventional multivessel percutaneous coronary intervention. Am J Cardiol. 2005;96:877-884. 
6. Fearon WF, Tonino PA, De Bruyne B, Siebert U, Pijls NH; FAME Study Investigators. Rationale and design of the Fractional Flow Reserve versus Angiography for Multivessel Evaluation (FAME) study. Am Heart J. 2007;154:632-636

7. Tonino PA, De Bruyne B, Pijls NH, et al. Fractional Flow Reserve versus Angiography for Guiding Percutaneous Coronary Intervention. N Engl J Med. 2009;360:213-224

8. Berger A, Botman KJ, MacCarthy PA, et al. Long-term clinical outcome after fractional flow reserve-guided percutaneous coronary intervention in patients with multivessel disease. J Am Coll Cardiol. 2005;46:438-442 .

9. Motoyama S, Masayoshi S, Harigaya H, et al. Computed tomographic angiography characteristics of atherosclerotic plaques subsequently resulting in acute coronary syndrome. J Am Coll Cardiol. 2009;54:49-57.

10. Jakó B, Benedek T, Suciu Zs, Benedek I. High Calcium Score Predicts Severity of the Culprit Lesions in Patients with Acute Coronary Syndromes. Acta Medica Marisiensis. 2014;59:298-301.

11. Ferrante G, Presbitero P, Whitbourn R, Barlis P. Current applications of optical coherence tomography for coronary intervention. Int J Cardiol. 2013:165:7-16

12. Maurovich-Horvat $\mathrm{P}$, Schlett $\mathrm{CL}$, Alkadhi $\mathrm{H}$, et al. The napkin-ring sign indicates advanced atherosclerotic lesions in coronary CT angiography. JACC Cardiovasc Imaging. 2012;5:1243-1252.

13. Diaz-Zamudio M, Dey D, Schuhbaeck A, et al. Automated Quantitative Plaque Burden from Coronary CT Angiography Noninvasively Predicts Hemodynamic Significance by using Fractional Flow Reserve in Intermediate Coronary Lesions. Radiology. 2015;276:408-415.

14. Ko BS, Wong DT, Cameron JD, et al. 320-row CT coronary angiography predicts freedom from revascularisation and acts as a gatekeeper to defer invasive angiography in stable coronary artery disease: a fractional flow reserve-correlated study. Eur Radiol. 2014;24:738-747.
15. Danad I, Raijmakers PG, Knaapen P. Diagnosing coronary artery disease with hybrid PET/CT: It takes two to tango. J Nucl Cardiol. 2013;20:874-890.

16. Meijboom WB, Meijs MF, Schuijf JD, et al. Diagnostic accuracy of 64-slice computed tomography coronary angiography: a prospective, multicenter, multivendor study. J Am Coll Cardiol. 2008;52:2135-2144.

17. Stuijfzand WJ, Danad I, Raijmakers PG, et al. Additional value of transluminal attenuation gradient in CT angiography to predict hemodynamic significance of coronary artery stenosis. JACC CardiovasC Imaging. 2014;7:374-386.

18. Li M1, Zhang J, Pan J, Lu Z. Obstructive coronary artery disease: reverse attenuation gradient sign at $\mathrm{CT}$ indicates distal retrograde flow - a useful sign for differentiating chronic total occlusion from subtotal occlusion. Radiology. 2013;266:766-772.

19. Nakanishi R, Matsumoto S, Alani A, et al. Diagnostic performance of transluminal attenuation gradient and fractional flow reserve by coronary computed tomographic angiography (FFR(CT)) compared to invasive FFR: a sub-group analysis from the DISCOVER-FLOW and DeFACTO studies. Int J Cardiovasc Imaging. 2015;31:1251-1259.

20. Ko BS, Wong DT, Nørgaard BL, et al. Diagnostic Performance of Transluminal Attenuation Gradient and Noninvasive Fractional Flow Reserve Derived from 320-Detector Row CT Angiography to Diagnose Hemodynamically Significant Coronary Stenosis: An NXT Substudy. Radiology. 2016;279:75-83

21. Choi JH, Min JK, Labounty TM, et al. Intracoronary transluminal attenuation gradient in coronary CT angiography for determining coronary artery stenosis. JACC Cardiovasc Imaging. 2011;4:1149-1157.

22. Wong DT, Ko BS, Cameron JD, et al. Transluminal attenuation gradient in coronary computed tomography angiography is a novel noninvasive approach to the identification of functionally significant coronary artery stenosis: a comparison with fractional flow reserve. J Am Coll Cardiol. 2013;61:1271-1279 